Artículos

\title{
LA RESOLUMEDIACIÓN DE CONFLICTOS EN EL ÁMBITO EDUCATIVO DE MANERA PARTICIPADA
}

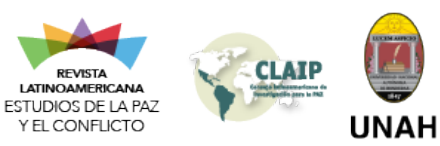

\author{
PARTICIPATORY RESOLU-MEDIATION OF CONFLICTS IN THE EDUCATIONAL AMBIT \\ RESOLUMEDIAÇÃO PARTICIPATIVA DE CONFLITOS NO ÂMBITO DA EDUCAÇÃO
}

\author{
Montañés Serrano, Manuel; Ramos Muslera, Esteban Andrés
}

\author{
Manuel Montañés Serrano \\ mms@soc.uva.es \\ Universidad de Valladolid, España \\ Esteban Andrés Ramos Muslera \\ esteban.ramos@gmail.com \\ Universidad Nacional Autónoma de Honduras, \\ Honduras
}

\author{
Revista Latinoamericana, Estudios de la Paz y el \\ Conflicto \\ Universidad Nacional Autónoma de Honduras, Honduras \\ ISSN: 2707-8914 \\ ISSN-e: 2707-8922 \\ Periodicidad: Semestral \\ vol. 2, núm. 3, 2021 \\ revistapaz@unah.edu.hn
}

Recepción: 28 Septiembre 2020

Aprobación: 03 Noviembre 2020

URL: http://portal.amelica.org/ameli/

jatsRepo/335/3351602006/index.html

DOI: https://doi.org/10.5377/rlpc.v2i3.10339

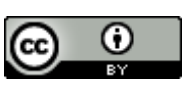

Esta obra está bajo una Licencia Creative Commons Atribución 4.0 Internacional.

Cómo citar / citation: Montañés, M. y Ramos Muslera, E.A. (2021). "La resolumediación de conflictos en el ámbito educativo de manera participada", Estudios de la Paz y el Conflicto, Revista Latinoamericana, Volumen 2, Número 3, 85-97. DOI: 10.5377/ rlpc.v2i3.10339
Resumen: Los modelos predominantes de mediación de conflictos en el ámbito escolar no pueden atender problemáticas sociales como el bullying, entre otras consideraciones porque la mediación requiere que sea voluntaria y que no sean cuestiones que puedan ser catalogadas como delictivas. En este artículo se realiza una razonada crítica tanto de los modelos principales de mediación escolar como de otra índole, se expone cómo el modo de atender las necesidades generan relaciones de cooperación y explotación (éstas últimas posibles precursoras de conflictos), se define el conflicto según la perspectiva sociopráxica, y se justifica y argumenta la necesidad de hacer partícipe al conjunto de redes y realidades grupales, presentes en el centro escolar, en un proceso cuya finalidad sea diseñar e implementar un Plan de convivencia escolar con el que anticiparse a los conflictos y cuando éstos están presentes, afrontar las problemáticas causantes de los mismos.

Palabras clave: Bullying, conflicto, constructivismo, mediación, sociopraxis.

Abstract: The predominant models of conflict mediation in the school environment cannot address social problems such as bullying, among other considerations, because mediation requires that it be voluntary and also that the issues are not classified as criminal. In this article a reasoned criticism of the main models of school mediation as well as others is made, how the way in which needs are met generates relationships of cooperation and exploitation (the latter being possible precursors of conflicts) is explained, conflict is defined according to the socio-praxical perspective and the need to involve the set of networks and group realities, present at the school, in a process aimed at designing and implementing a Plan for school coexistence with which to anticipate conflicts and, when they are present, to deal with the problems that cause them.

Keywords: Bullying, conflict, constructivism, mediation, sociopraxis.

Resumo: Os modelos predominantes de mediação de conflitos no ambiente escolar não podem abordar problemas sociais como o bullying, entre outras considerações porque a mediação exige que seja voluntária e que não sejam questões que possam ser categorizadas como criminosas. Este artigo faz uma análise crítica dos principais modelos de mediação escolar bem como de outros modelos, explica como a forma de atender às necessidades 
gera relações de cooperação e exploração (sendo este último possível precursor de conflitos), define o conflito de acordo com a perspectiva sócio- prática, e justifica e argumenta a necessidade de fazer com que o grupo de redes e realidades grupais, presentes na escola, participem de um processo cujo objetivo é desenhar e implementar um Plano de convivência escolar com o qual antecipar os conflitos e, quando presentes, lidar com os problemas que os causam.

Palavras-chave: Bullying, conflito, construtivismo, mediação, sociopraxis.

\section{Extended Abstract}

Pre-eminent models of mediation in the educational ambit cannot go beyond the resolution of small disputes, without tackling major problems of social concern, such as bullying or other types of violence in general, among other reasons because in these models, conflicts are dealt with on an individual basis, the people involved have to voluntarily request mediation and the actions under analysis cannot be classified as a crime.

Conflicts are not of an individual nature, but are rather group-based, involving not only the people directly intervening. Therefore, for the problems that gave rise to the conflict, or the more or less violent actions to be satisfactorily addressed, the multiple networks and group realities present in the school must be involved. This requires knowing the type of relationships that the different group realities have with each other, as well as researching how the other is perceived and built. To this end, a participatory process has to be activated to elaborate and implement a Plan for Coexistence in which the recognition of the Other is debated and reflected upon, with the aim of building a "we", that is, an inclusive "other", in which each one, being different, forms a group with others. In this article, the main phases and contents that the participatory process should contemplate are enunciated.

The proposal formulated here with the neologism of resolumediation unites in the same syntagma the resolution and the mediation in conflicts. Strictly speaking, insofar as it deals with more or less violent problems and is carried out without waiting for the people involved to request intervention, it is not a proposal for mediation, but to the extent that it resolves (or/and anticipates) the conflict in a participatory manner it can be considered a form of mediation, among other issues because the fact of participating in the process is itself a form of social mediation.

\section{Notas DE AUTOR}

\footnotetext{
Manuel Montañés Serrano es licenciado y doctor en CCPP y Sociología. Es Profesor Titular en la Universidad de Valladolid (Campus de Segovia). Sus publicaciones de este año son las siguientes: "El asociacionismo y la participación vecinal luchando por la vivienda, haciendo ciudad", Hábitat y Sociedad, n 13, pp. 287-298, 2020; "Investigación y Formación nacional e internacional en la Universidad Nacional Autónoma de Honduras", Utopía y praxís Latinoamericana, no 90, pp. 264-28, 2020; Estrategia y metodología participativa del modelo ciudadanista de planificación y diseño de políticas públicas. En Quintero, M.C. y Granja, L. C. (Eds.), Políticas públicas: reflexiones y experiencias latinoamericanas. Cali (Colombia): Editorial Universidad Santiago de Cali; “El 15-M: Origen, características, fortalezas y debilidades, e influencias y trascendencia”, Estudios de la Paz y el Conflicto. Revista Latinoamericana. Vol. 1, n 1, pp. 59-73, 2020.

Esteban A. Ramos Muslera es licenciado en Ciencias Políticas por la Universidad Complutense de Madrid, Magister en Investigación Participativa para el Desarrollo Local por la misma universidad y Doctor en Ciencias Políticas por la Universidad de Valladolid. Actualmente, es Coordinador del Área de Paz del Instituto Universitario en Democracia, Paz y Seguridad (IUDPAS) de la Universidad Nacional Autónoma de Honduras (UNAH), Council member de la International Peace Research Association (IPRA), y Secretario General del Consejo Latinoamericano de Investigación para la Paz (CLAIP). Sus dos últimas publicaciones son: "La participación del estudiantado en el diseño curricular de la Educación para la Paz Transformadora" (2019), en OBETS. Revista de Ciencias Sociales, 14 (2), 437-470; e, "Investigación y Formación nacional e internacional en la Universidad Nacional Autónoma de Honduras”, Utopía y praxís Latinoamericana, nº 90, pp. 264-28.
} 


\section{INTRODUCCIÓN}

La mediación en la resolución de conflictos en el ámbito educativo, en los que se encuentran implicados jóvenes-adolescentes, de acuerdo con los modelos preeminentes no puede ir más allá de la resolución de pequeñas disputas, sin que se puedan abordar grandes problemas que preocupan socialmente, como es el bullying -entendido como los "actos intencionales reiterados en el tiempo que causan daño, hieren o incomodan a otra persona en el marco de relaciones asimétricas de poder" (di Napoli, 2016: 63)-y la violencia de más o menos intensidad, en general. El acoso escolar y la violencia que se produce en el ámbito educativo requieren implementar un modelo de resolumediación de conflictos en el que la comunidad educativa participe en el diseño e implementación de planes de convivencia escolar.

Los modelos de mediación al uso no pueden encarar problemáticas sociales como el bullyng, entre otras razones porque en estos modelos se atiende los conflictos de manera individual, las personas implicadas han de solicitar voluntariamente la mediación y las acciones objeto de análisis no pueden ser calificadas como delito (Prada y López, 2005). Es por ello que la propuesta que aquí se formula une en el sintagma la resolución y la mediación en los conflictos. La propuesta stricto sensu no es una propuesta de mediación, pero en tanto resuelve (o/y se anticipa al) el conflicto de manera participativa puede considerarse un modo de mediación.

En las páginas que siguen se hará una crítica razonada de los principales modelos de mediación, se expondrán los argumentos teóricos y conceptuales de la perspectiva sociopráxica de la realidad social, definiéndose el conflicto de acuerdo con este enfoque, se da cuenta de cómo la presencia de posibles conflictos depende del modo en que son atendidas las necesidades, y, por último, se enunciarán los rasgos principales de la metodología con la que construir participativamente planes de convivencia en el ámbito escolar.

\section{MODELOS}

Según Torrego (2001) existen tres grandes modelos de resolución de conflictos en el ámbito educativo: el Normativo punitivo-sancionador, el Interpersonal-relacional, y el Integrador.

\subsection{Normativo, punitivo-sancionador}

Este modelo se enmarca en una perspectiva reglamentista de las prácticas y conductas sociales. Se recurre a los reglamentos de los centros, en donde se señalan los derechos, deberes, faltas y sanciones, para establecer las normas de convivencia. Se podría decir que el reglamento es el que media para que no se produzcan prácticas que generen malestar. Y cuando así acontece, la sanción a la persona que trasgrede la norma se convierte en una forma de reparar a quien ha sido agraviada.

Este modelo obvia que la existencia de sanciones no garantiza per se que no se transgredan las normas establecidas. Y, asimismo, obvia que la aplicación de sanciones no necesariamente implica que el conflicto se regule satisfactoriamente, pues cabe la posibilidad de que las sanciones generen el efecto inverso al pretendido, no solo no se resuelva, sino que se agrave aún más el conflicto: la persona agraviada puede sufrir represalias por parte de las personas sancionadas, de sus respectivos entornos o/y de otras más, al hacerla responsable de las sanciones que la dirección del centro educativo impone.

La aplicación de normas en las que no se ha participado en su elaboración por órganos de los que no se forma parte, puede implicar un rechazo -con la consiguiente acorde respuesta- no solo de la persona sancionada sino de otras muchas más.

La participación del conjunto de la comunidad educativa tanto en la elaboración de las normas de convivencia (y las sanciones correspondientes) como en los órganos vigilantes y ejecutores de las mimas, sin 
Revista Latinoamericana, Estudios de la Paz y el Conflicto, 2021, 2(3), Enero-Junio, ISSN: 2707-89...

duda, contribuirá a la resolución de los problemas asociados al conflicto de manera satisfactoria. Entre otras cuestiones porque el hecho en sí mismo de participar en el proceso es una forma de mediación social.

\subsection{Modelo interpersonal relacional}

Este modelo puede ser considerado como el protomodelo de la mediación, entendiéndose como tal,

"un proceso confidencial, voluntario, y estructurado de gestión y resolución de los conflictos que sirve para que dos partes que estén inmersas en algún conflicto entre sí, consigan solucionarlo de una forma satisfactoria, aceptando la ayuda de una persona mediadora, profesional, experta y debidamente formada que tiene como características principales la de ser imparcial y no imponer acuerdos, pero dirigiendo a las partes a la consecución de los mismos y al logro de su cumplimiento, siendo éstos equilibrados y equitativos" (Diego y Guillen, 2006: 19).

Mediante el diálogo entre las personas implicadas se persigue que éstas modifiquen sus relaciones atendiendo a las tres erres que acuñara Galtung (1998): reparación por el daño causado, reconciliación de las partes, y resolución del conflicto subyacente.

Este modelo no tiene en cuenta que los conflictos, como señala Calderón (2009), no son de carácter individual, en los mismo están, más o menos presentes, de manera más o menos activa, diferentes realidades grupales. De ahí la importancia de implicar en el proceso de construcción de planes de convivencia al conjunto de redes y realidades grupales presentes en el ámbito educativo. De este modo, quien ejerce la mediación contará con una amplia legitimidad para ejercer su labor.

\subsection{Modelo integrador}

Este modelo integra los dos anteriores. Se propicia la comunicación entre las partes implicadas en el conflicto para que resuelvan las problemáticas asociadas al conflicto satisfactoriamente, recordando, de manera explícita o implícita que, si las conversaciones no tienen su fruto, se recurrirá al reglamento del centro para establecer las medidas que se adoptaran.

Para no reproducir el modelo punitivo, distintas son las estrategias a las que se puede -y se suele- recurrir de manera complementaria, propias éstas de otros modelos no estrictamente relacionados con la mediación en el ámbito educativo, como son el Tradicional-lineal o de Harvard, el Transformador, y el Circular narrativo.

\subsection{Modelo Tradicional-lineal o de Harvard}

Aunque este modelo no es un modelo de mediación, sino de negociación asistida, por su influencia en las experiencias y prácticas de la mediación, también en el ámbito educativo, es preciso considerar sus rasgos principales (Cornelius y Faire, 1995;Díez y Tapia, 1999;Folger y Jones, 1997; Muldoon, 1998). Siguiendo a Fisher y Ury (1981), la aplicación de este modelo requiere que las personas implicadas en el conflicto separaren sus posiciones del análisis de la problemática objeto del malestar. Se ha de procurar que cada una observe la pobremática teniendo en cuenta tanto sus intereses y necesidades como las de la otra parte, de tal modo que cooperen en la elaboración de una propuesta que satisfaga a ambas partes. A tal fin, la mediación ha de indagar sobre el BATNA (Brest Alternative To Negotiated Agreement), esto es, los puntos, aspectos, cuestiones, grado, intensidad, contenido, etc. que cada parte considera el límite máximo que está dispuesto a ceder (o ser más o menos flexible), y, asimismo, ha de procurar que las partes tengan en cuenta los criterios formales relacionados con la convivencia (derechos, obligaciones y sanciones) para que sopesen la pertinencia de llegar a un acuerdo negociado como alternativa a un dictamen formal, que pudiera no satisfacer a ninguna. 


\subsection{Modelo Transformador}

Siguiendo a Bush y Folger (1996), este modelo pone el foco en el proceso comunicativo mediante el cual las partes implicadas han de lograr un conocimiento recíproco. Uno conoce al otro y el otro conoce al uno. Las personas elaboran sus propuestas a tenor de una comprensión más clara y seguras de sí mismas, de cada parte y de la naturaleza de los temas que generan malestar. En esta acción comunicativa, la mediación ejerce un papel clave como facilitador de la misma. A tal fin se recurre, entre otros mecanismos, dispositivos y herramientas, a la escucha activa -esto es, no solo prestar atención a la dimensión referencial del lenguaje sino también a la estructural y afectiva-emotiva-, a psicodramas (en los que se juega a intercambio de roles), a la exposición de resúmenes, a preguntas de verificación, y a técnicas como la del "espejo", consistente en reenviar las afirmaciones y ver la actitud y comportamiento que genera.

\subsection{Modelo circular narrativo}

Este modelo, acuñado por Sara Cobb (1993: 1997), como los dos anteriores, recurre a la comunicación como herramienta con la propiciar la mediación. Se fundamenta en la dimensión pragmática del lenguaje (esto es, en la capacidad del hacer y no solo del decir del lenguaje) y en el constructivismo social, según el cual los seres humanos no damos cuenta de una realidad externa y prexistente, sino que la construimos. Se sostiene que las personas construyen relatos con los que estructuran sus experiencias de manera coherente, desechando los aspectos que pueden poner en peligro el relato elaborado, seleccionando e insertando otros que lo reafirmen, generándose así un proceso circular retroalimentador. Es por ello que se insta a que se reflexione sobre lo que se dice, sobre lo que uno dice de sí mismo, del otro, y sobre la problemática conflictual. A diferencia del modelo de Harvard, en este caso, se busca potenciar las diferencias entre las narrativas que construyen las partes con la intención de generar alternativas posibles, que, cual estructuras disipativas (Prigogine y Stengers, 1985), ordenen a partir de la diferencia y el caos. El trabajo fundamental de la mediación es generar una historia alternativa a partir de las narrativas de las partes para acometer su devolución con la intención de facilitar la observación, reflexión, debate y retroalimentación de la historia, y, finalmente, establecer un acuerdo. La originalidad de este modelo recae en la construcción y reconstrucción dialógica de la narrativa alternativa para así poder formular una propuesta superadora.

\subsection{Valoración de los modelos}

Estos modelos no contemplan la dimensión fundamental de los conflictos en general, ni los que se generan en el ámbito educativo especialmente. Los conflictos, como se ha dicho antes, no son de carácter individual sino que son grupales. El modelo Ecosistémico (Parkinson, 2012) sí contempla la dimensión grupal, al incluir a los hijos e hijas y no solo a los cónyuges en la mediación en el ámbito familiar; si bien, en la propuesta que aquí se formula, la dimensión holística e integral no solo demanda la participación de uno o varios núcleos sino, dado que en los conflictos no solo se encuentran implicadas las personas intervinientes, la participación de todas las realidades y redes grupales en el proceso de construcción de los planes de convivencia escolar. En todo caso, hay aspectos de los modelos enunciados que ayudan a comprender los conflictos y el modo de afrontarlos, como es la comunicación como herramienta y objeto de análisis, la cooperación en la construcción de propuestas superadoras con las que mejorar la convivencia, la necesidad de reflexionar sobre el reconocimiento del otro -más bien aceptación de la otredad-, y la pertinencia de prestar atención al modo en el que se construye el conflicto y a la dimensión pragmática del lenguaje en su construcción. Si bien, la perspectiva constructivista que se ha de considerar no ha de ser de tipo idealista sino materialista, de la que se dará cuenta en el epígrafe siguiente. 
Además, como apunta Redorta,

“para entender mejor la importancia de tener planteamientos claros en lo que respecta a la obtención de resultados detallamos aquellos factores que, a nuestro juicio, deben ser tenidos en cuenta para cualquier tipo de conflicto:

a) Los factores que resultan permanentes en la relación entre las partes. Por ejemplo: la edad, el género, el rol, la historia, etc.

b) Los factores que derivan de la relación que las partes sostienen. Por ejemplo, dependencia, familia, vecindad, afinidad política, etc.

c) Las características del conflicto. No se tratan igual los conflictos vinculados a la autoestima, que los conflictos de poder, por poner un ejemplo.

d) Las características del gestor o gestores del conflicto. La suma de habilidades y recursos es muy importante y afecta al resultado final" (Redorta, 2020: 95).

\section{PERCEPCIÓN RELATIVA Y REALIDAD CONSTRUIDA}

Hay múltiples ejemplos en nuestra vida cotidiana y otros tanto diseñados ad hoc en los que se aprecia nítidamente la percepción relativa de la realidad, como en la imagen $\mathrm{A}$, en que se puede observar una mujer joven o una anciana, o la $\mathrm{B}$, en la que se puede observar dos caras de perfil mirándose o un candelabro. El fondo o la forma no dependen de lo observado sino del sujeto observador.

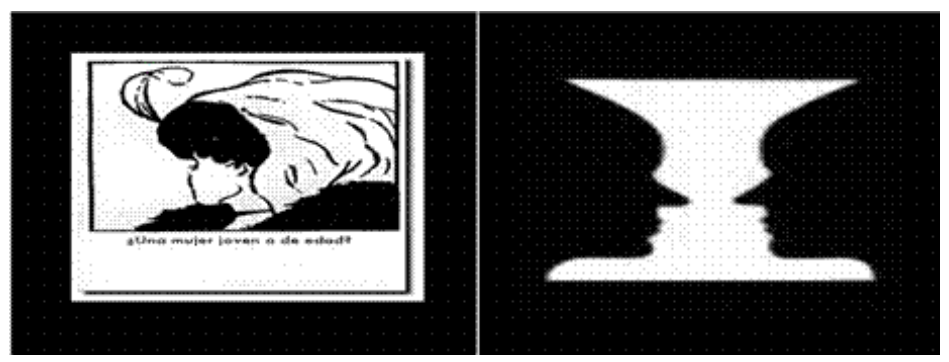

FIGURA 1

Imagen A) mujer joven y anciana. Imagen B) dos caras y un candelabro

Imagen A: Ilustración creada por W.E Hill, publicada en el año 1915 en la revista

estadounidense Puck con el título "Mi mujer y mi suegra". Imagen B: Autoría desconocida.

De ambos ejemplos se dice que se puede observar dos realidades, tanto una como otra, o una u otra. Si bien, en realidad no se puede observar a una mujer joven o a una anciana, ni un candelabro, ni caras mirándose, vemos la representación de personas u objetos.

Accedemos a la realidad representándonosla. La observación humana, y, por ende, la conducta que se desprende de la misma, no es pasiva, es un acto creativo. Los seres humanos no vemos árboles, montañas, ríos o casas, sino que inferimos sentido a los estímulos electromagnéticos recepcionados a los que nuestro cerebro, que no nuestros ojos, infiere sentido. Si no fuese así, si la realidad no fuese producida por el sujeto, si la realidad fuese la que ven nuestros ojos, las personas ciegas y las que no lo son vivirían en mundos paralelos. Si habitamos en un mundo inteligiblemente compatible es porque unos y otros compatibilizamos los sentidos inferimos a los estímulos recepcionados por las terminales nerviosas. Cuando no se compatibilizan las categorías socioculturales elaboradas, aunque no se tengan problemas ópticos, se ve de distinta manera. A diferencia del apóstol santo Tomás, no creemos porque veamos, sino que vemos porque creemos. Cuenta Castaneda que estando caminando por la selva de México, su maestro, Don Juan, se detenía cada cierto tiempo y le decía: "¡Mira, mira lo que hay ahí! ¿Lo vistes?” A lo que él, invariablemente contestaba que no lo había visto. Tras un período de reflexión, Don Juan, al darse cuenta de la situación, exclamó: "¡Ahora entiendo cuál es tu problema! Tú no puedes ver lo que no puedes explicar. Trata de olvidarte de tus explicaciones y comenzarás a ver" (Castaneda, 1978). 
El ser humano es y vive en un mundo cultural. No es ya solo que disponga de la capacidad cultural para afrontar el devenir sino que es y vive en un mundo de representaciones de representaciones que procura compatibilizar, no compartir, con otros seres humanos, pues compartir y compatibilizar no es lo mismo. Compartir es participar de una misma realidad, compatibilizar es hacer que la realidad de uno encaje con la realidad de otro u otros. Como señala von Glasersfeld:

"hablar de significados compartidos es un sin sentido puro [...]: no he construido el lenguaje como no he construido esta mesa, pero me he adaptado a la mesa no atravesándola. Me he adaptado al lenguaje que existe construyendo mis significados de manera tal que encajen en mayor o menor medida con los significados de los otros. Pero "encaje" no es equivalencia. "Compatible" no quiere decir "igual", simplemente significa que no causa problema" (Glasersfeld, 1994: 138).

Los seres humanos no compartimos con nadie ninguna realidad porque no hay realidad preexistente alguna, y, sin embargo, no tenemos ninguna duda en afirmar que vemos realidades objetivas, universales, externas y preexistentes a uno. Ello se debe a la capacidad reflexividad de representarnos representándonos la realidad que nos representamos, dicho para que se entienda de manera más clara, gracias a que nos vemos viendo la realidad que vemos - esto es, tenemos consciencia-, podemos ver a otros viendo la realidad que vemos - no solo mirando-, generándose, de este modo, el efecto de realidad objetiva.

Toda realidad social es construida, si bien, ésta no es fruto del capricho de cada cual, como desde un constructivismo idealista abstracto pudiera considerarse (en el que ego subjetivo y su conciencia fuese lo único real) sino que es de índole materialista. Son las necesidades las que nos impelen a realizar unas u otras prácticas, a cuyos estímulos generados le inferimos particulares e intransferibles sentidos que, si se quiere seguir viviendo, se han de compatibilizar en las redes en las que estamos obligados a participar. No necesariamente con todas y con todos los componentes de las redes, pero tampoco puede no hacerse con nadie. Se puede decir que se ha de compatibilizar con quien se quiera de entre los que se pueda.

Los seres humanos no vivimos en un vacío existencial. Vivir conlleva acoplamientos perceptivomotores con el medio y con quienes habitan en el mismo, generándose efectos, cuya valoración propicia la construcción de una u otra realidad, y, por ende, una u otras acciones. Las prácticas modifican el medio y la valoración de los efectos producidos hace que la realidad social construida sea de un modo u otro, al tiempo que nos modificamos al ser seres sociopráxicos que nos transformamos en la acción de transformar. Los demás seres vivos únicamente se degradan en el proceso entrópico de transformación de la energía pero no se transforman cognitivamente en la acción de transformar.

\section{EL CONFLICTO DESDE LA PERSPECTIVA SOCIOPRÁXICA}

El conflicto, como toda realidad, es socialmente construido en el proceso de compatibilización de realidades producto de la concatenación de acciones-valoraciones- respuestas que en el marco de la acción relacionalcohabitacional humana se produce. Y como toda realidad socialmente compatibilizada por múltiples realidades grupales, se ha de poder observar como una sustantiva realidad.

Tomando como referencia la definición de Montañés y Ramos (2012), se puede entender el conflicto como una sustantiva realidad en la que los efectos de las acciones de unos seres humanos generan malestar en otro u otros, considerando el/los afectado/s que los sujetos de la acciones emprendidas saben que las misma (las acciones emprendidas) generan malestar en los primeros, impeliendo a éstos a emitir respuestas, cuya valoración, por parte de los egundos sujetos, genera algún grado de inquietud o/y malestar en los mismos, impeliéndoles asimismo a realizar acciones, de la misma u otra naturaleza, generando malestar en los primeros. Encadenándose acciones de un o unos y otros, perdurando en el tiempo las acciones y el consiguiente malestar, quedando implicadas en este proceso diversas redes y realidades grupales.

Préstese atención a los componentes de la definición: 
1. Analizar, y, por ende, resolver el conflicto, requiere que éste sea considerado una sustantiva realidad y no como una queja o malestar de unos sujetos, aunque la queja pueda ser el llamado para afrontarlo.

2. Son acciones humanas, lo que implica que deberá achacarse a otro u otros seres humanos el malestar padecido. Si, por ejemplo, una persona es mordida por un perro, es probable que el mordisco le genere un malestar terrible, pero nunca un conflicto con el perro. Si acaso, se podría llegar a generar un conflicto con el amo del perro, si se cumplieran otros requisitos.

3. Para que exista conflicto no es suficiente con que alguien acometa acciones que generen malestar en otro u otros sujetos, es necesario a) que los sujetos que realizan las acciones sepan que se les hace/ $\mathrm{n}$ responsable/s de las acciones, $\mathrm{y} \mathrm{b}$ ) que la persona/as que padecen el/os agravios emprenda/ $\mathrm{n}$ alguna/s acciones, por leves o incontundentes que sean, en contra de los que han realizado la/s práctica/s generadora/s de malestar. Por tanto, toda acción violenta, sea física, psicológica, de otra índole, o simbólica -entendiéndose por tal, la asunción como algo natural y necesarias conductas y prácticas sociales que otros o en otro tiempo y lugar no se consideran aceptables- no puede considerarse un conflicto. Otra cuestión es que pueda ser la antesala de este.

4. Tanto las acciones que generan malestar como las respuestas a las mismas, se tienen que concatenar y perpetuar en el tiempo. Si no es así más que conflicto se está en presencia de acciones vandálicas o más o menos asociales.

5. Para que se pueda hablar de conflicto social, es necesario que una o unas redes (de parentesco, personales, vecinales, generacionales, étnica, de clase, de género, de hábitat, etc.) estén implicadas. En los conflictos en los que aparentemente solo está implicado el agresor y la victima hay toda una serie de redes y realidades grupales que sostienen la agresión -ya sea participando en las misma o apoyando, jaleándola, festejándola, aplaudiéndola, estimulándola, etc.- y hay diferentes sujetos que la padecen, aunque unos la padezcan con más o menos intensidad, respondan con más o menos contundencia, y sea ésta más o menos organizada por más o menos personas.

\section{Necesidades y satisfactores}

Las acciones que generan malestar se debe a que el modo de atender las necesidades sociales no es de manera sinérgica, esto es, no son atendidas las necesidades propiciando que otras de unos y de otros y de otras sean también atendidas.

Las necesidades humanas son necesidades socioculturales. El ser humano no tiene necesidades en tanto que ser biológico y necesidades socioculturales en tanto que ser humano, sino que tiene necesidades en tanto es un ser cultural. El modo que tenemos de representarnos el mundo, y a quien en él habitan, nos impele a definir unas u otras necesidades, así como el modo de atenderlas. Decir que hay necesidades primarias atemporales y universales es caer en la abstracción ya que éstas se han de concretar en medios y modos concretos. En distintas épocas y lugares los diferentes grupos humanos han articulado sus necesidades a escala humana más allá de la dimensión fisiológica. No hay constancia de la existencia de un grupo humano cuyos miembros se hayan limitado a mantener las constantes vitales. Los seres humanos necesitamos hidratos para seguir viviendo, pero en tanto que seres culturales hemos de concretar la forma, el cómo, el dónde, con quién, el cuándo y el qué hemos de ingerir para conseguirlos. Las formas que tengamos de dar respuestas a estas preguntas articularán una u otras necesidades. Todos los seres humanos que habitamos y han habitado el planeta procuramos vivir en tanto que seres humanos con necesidades humanas. Otra cuestión son los satisfactores, término acuñado por Max-Neeff, Elizalde y Hopenhayn (1986). Unos u otros han estado presente en distintos momentos, diferentes épocas o cultura. La lectura rápida que se ha hecho de los escritos de estos autores ha propiciado que se considere el término satisfactor como sinónimo de medio con el que atender una necesidad. Sin embargo, no son medios o recursos, sino que han de ser entendidos como modos de atender las necesidades. Son 
formas de Ser, Tener, Hacer y Estar conducentes a la actualización de las necesidades. Según estos autores hay nueve necesidades axiológicas: subsistencia, protección, afecto, entendimiento, participación, ocio, creación, identidad, y libertad. El cruce de estas necesidades con las categorías existenciales o modos de experiencia de Ser, Tener, Hacer, y Estar, según el satisfactor que se aplique serán atendidas de una u otra forma.

Cinco son, según los autores citados, los satisfactores que se pueden aplicar: a) Violadores y destructores: el modo que tienen estos satisfactores de atender una necesidad de uno es a costa de impedir o dificultar que puedan atenderse otra necesidad y que otros seres puedan también atender las suyas, como cuando un grupo de alumnos se apropia de la mayoría del espacio del recreo para jugar al fútbol a costa de que las alumnas y muchos otros alumnos no tengan más remedio que restringir sus movimientos al reducido espacio que les queda; b) Pseudosatisfactores: son los que crean una falsa sensación de haber cubierto una necesidad, como cuando, siguiendo con el ejemplo anterior, se les dice a las alumnas que se sumen al juego balompédico; c) Inhibidores: esto atienden en demasía una necesidad impidiendo u obstaculizando que se puedan atender otras, como cuando el fútbol es la práctica que ocupan la mayoría del espacio todos los días y durante todo el tiempo del recreo; d) Singulares: son los que atienden una sola necesidad sin tener en cuenta la suerte que pueden correr las demás, como cuando se destinan la práctica totalidad de los recursos a acondicionar el patio para que se pueda jugar al fútbol sin sopesar otras necesidades que asimismo tendrían que ser atendidas, y e) Sinérgicos: son los que atienden una necesidad estimulando y contribuyendo a que sean atendidas las demás necesidades, como cuando se distribuyen tiempos y espacios para que todos y todas puedan hacer un uso equitativo del espacio.

Cuando el modo de atender las necesidades es mediante satisfactores violadores o destructores, se genera malestar, y, por ende, el conflicto es previsible que tenga lugar, y, también, aunque sea más fáciles de revertir, cuando se recurre a otros que no sean sinérgicos.

Para que sean atendidas las necesidades de modo sinérgico, se convendrá que no será apelando a la buena voluntad de quienes las atienden de modo violento o destructor para que dejen de hacerlo y se decanten, sin solución de continuidad, inopinadamente, por un modo sinérgico. Solo dejarán de hacerlo cuando no puedan seguir haciéndolo o vean que obtienen más beneficio al optar por un modo sinérgico que por un modo violador o destructor. Los acosadores, póngase por caso, no dejarán de acosar simplemente porque se apele a su buena voluntad. Y cabe tener en cuenta que el recurso a la sanción para que modifiquen la conducta no siempre logra el objetivo pretendido, pudiéndose generar, como se ha dicho antes, el incremento de las acciones acosadoras por las personas sancionadas o/y por otras cercanas a las mismas.

En el acoso escolar están implicadas múltiples realidades grupales, las que directamente ejercen la violencia física, psíquica, mental o simbólica, las que jalean las acciones, las que las padecen, y las que son conocedoras de las mimas y se mantienen al margen, bien por miedo a convertirse en próximas víctimas, o porque consideran que, de algún modo, quienes lo padecen son merecedores de ello, o porque mientras no se vean afectadas directamente no consideran que les incumba y, por tanto, no es un problema suyo. Para acabar con el acoso, se ha de lograr que disminuya el número de quienes lo ejercen -hasta logra que no haya ninguno-, no haya quienes los jaleen y que quienes se muestran al margen se impliquen en defensa de quienes lo padecen, e, incluso, que estos últimos se vean con fuerzas para hacer afrontarlo. En resumen, se ha de conseguir la máxima freireana (Freire, 1970) de unir los afines con los diferentes, incluso con los ajenos, para oponerse a los antagónicos. Ello requiere que las diversas realidades grupales debatan, reflexionen y formulen colectivamente medidas sobre el acoso escolar, el cual se fundamenta en no reconocer al otro como un nosotros, siendo como somos todos y todas otros y otras de otros y otras por ser bajito, alto, obeso, flaco, tener diferentes capacidades físicas, psíquicas o mentales, homosexual, lesbiana, transexual, asexual, intersexual, gitano, migrante, mujer, pobre, o por vestir según se considere, o llevar el pelo de una manera u otra o no tenerlo, etc.

La implicación de los y las indiferentes en la resolución satisfactoria de la problemática logra que disminuya la base que sustenta a los acosadores, y, asimismo, la aparición de naturales mediadores, ya que al no encontrarse segregadas las redes sino que, en mayor o menor medida, hay quienes pertenecen a diferentes 
redes, habrá quienes tenga contactos con diferentes realidades grupales. Esto mediadores pueden propiciar la reflexión y la exposición del relatos de los acosadores que, tal vez, puedan actuar de manera violenta al formar parte de la cultura de la violencia, la cual le lleva a agredir antes de ser agredidos y a ser violentos como respuesta de la violencia estructural o/y personal que padecen.

Ello ayudará al diseño de un Plan de Convivencia Escolar. En su confección ha de participar el conjunto de la comunidad educativa. Al tan fin, se ha de diseñar e implementar un proceso participativo. A continuación, se enuncia las fases y los contenidos principales del mismo.

\section{PROCESO PARTICIPATIVO CON EL QUE DISEÑAR EL PLAN DE CONVIVENCIA ESCOLAR}

Como se puede apreciar en la figura 2, el proceso que se propone se estructura en cuatro fases: Conversación inicial, Conversación en las redes, Dialógica informativa, y Conversación proyectiva.

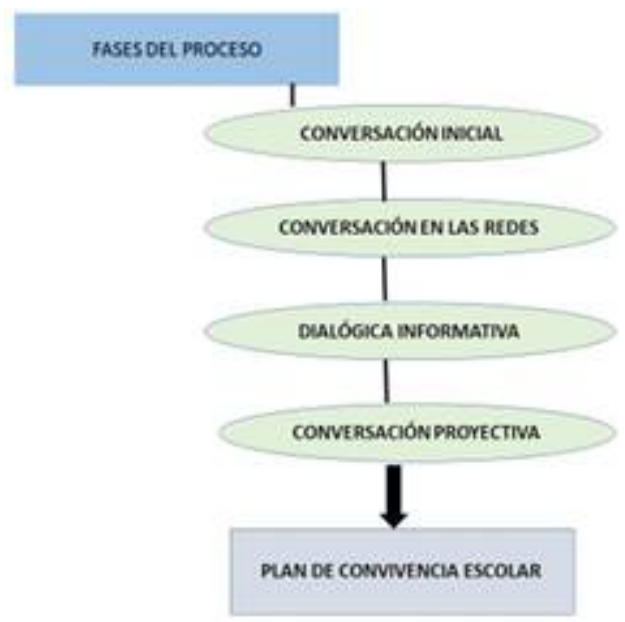

FIGURA 2

Fases del proceso de diseño del Plan de Convivencia Escolar

Elaboración propia

Exponer pormenorizadamente y desarrollar el contenido de cada una de las fases superaría la razonable extensión y el propósito de un artículo. Aquí se expondrán los aspectos más sustanciales y significativos de cada una de ellas. En otras publicaciones los autores de este artículo han tratado de forma detenida y profusa los contenidos de cada fase, así como las técnicas e instrumentos que se recomienda emplear en cada una de las mismas: (2006, 2009 y 2012), Montañés y Martín (2017) y Ramos (2015, 2019).

\subsection{Conversación inicial}

En esta fase se constituye la Comisión de Seguimiento, formada por responsables del equipo directivo, por las asociaciones de padres y madres y por las de alumnado, si la hubiera, y en el caso en que no fuera así por algunos representantes. Como su nombre indica, realiza el seguimiento, supervisa y reorienta el proceso, siendo la búsqueda del consenso la guía que oriente la toma de decisiones. Asimismo, se crearán las comisiones de trabajo que se estimen oportunas. Se constituye el grupo motor, formado por el equipo técnico y por los colaboradores que participan con más o menos intensidad en el día a día. Su participación es de especial utilidad para el desarrollo del proceso, ya que proporcionarán información contextual, ayudarán a elaborar los sociogramas, proporcionarán y realizarán contactos y favorecerán la contrastación de ideas al colaborar con cierta asiduidad en el proceso. Se diseña y se pone en marcha un plan de comunicación y dinamización social 
con el que motiva al conjunto de la comunidad educativa a participar en el proceso, y asimismo, se realiza un autodiagnóstico participativo. Para tal fin se pueden realizar encuentros participativos de creatividad social (EPCS) en las aulas y entre el profesorado en los que haciendo uso de la Matriz DAFO, en la que en un mismo plano, se vean las Debilidades, Amenazas, Fortalezas y Debilidades, y asimismo se formule lo que se considera que se ha de hacer para anular, o, al menos reducir la debilidades, eludir o amortiguar las amenazas, mantener y ampliar las fortalezas y aprovechar las oportunidades.

\subsection{Conversación en las redes}

En esta fase se ha de confeccionar un sociograma en el que se visualice el tipo de relaciones-afinidad, oposición, complementariedad, ajenidad, etc.- que mantienen entre sí las diversas realidades grupales presentes en el centro educativo. Y, asimismo, el diagnóstico de la fase anterior ha de complementarse con el conocimiento de los argumentos, razones e ideas y la base en la que descansa la construcción sobre el centro, sus malestares y conflictos posibles o manifiestos. Para ello, se han de realizar entrevistas y grupos de discusión que den cuenta de las posiciones discursivas al respecto. Esto es, que den cuenta tanto de las problemáticas sociales como el modo de afrontarlas. También se puede recurrir a la Matriz de escenarios presentes y futuros, consistente en cuatro cuadrantes, los dos de la izquierda referidos al futuro y los dos de la derecha al presente, los de arriba a los aspectos negativos y los de abajo a los positivos. En el cuadrante de arriba a la izquierda se enuncian los aspectos que no están presentes y no se quiere que tengan lugar; en el segundo cuadrante, en el de arriba a la derecha, los aspecto que en la actualidad son valorados negativamente; el tercer, en el cuadrante de abajo a la derecha, los que en el presente son valorados positivamente; y el cuarto, en el cuadrante de abajo a la izquierda, los aspectos positivos que no están presentes y se quisiera que estuvieran.

\subsection{Dialógica informativa}

En esta fase tiene lugar el debate y la reflexión sobre la información obtenida en las fases anteriores. Se ha de poner los medios para que se piense sobre lo pesando y se diga sobre lo dicho o por decir, esto es, se reflexione sobre qué, de qué, de quién, por qué, para qué se dice lo que se dice o no se dice lo que dice, y asimismo, se ha de recurrir a sociodramas y técnicas del espejo, entre otras, al fin de potenciar la empatía que ayude al reconocimiento de la otredad.

\subsection{Conversación proyectiva}

La reflexión de la fase anterior ha de servir para la construcción colectiva del Plan de Convivencia Escolar. El cuál ha de contemplar, al menos, una estructura organizativa y de gestión, normas de convivencia, actividades y tareas a realizar, recursos económicos, humanos, materiales con los que ve a contar y, asimismo, un sistema evaluación y monitoreo con el que conocer si la implementación del mismo es y está siendo eficaz y eficiente.

\section{CONCLUSIONES}

El modo en que son atendidas las necesidades genera relaciones de cooperación o de explotación, en este último caso, unos disfrutan a costa del malestar de otros, propiciándose la aparición de conflictos sociales de diferentes índole y magnitud.

El modo de resolver los conflictos depende de la concepción que se tenga de la naturaleza de la realidad social. Sea ésta objetiva, relativa o construida. 
Si la realidad fuese objetiva, se analizarían los comportamientos de las personas implicada en los conflictos sin necesidad de indagar sobre sus percepciones y valoraciones. La mediación en la resolución de conflictos seria del tipo reglamentista, valorándose los objetivos hechos según las normas, derechos, obligaciones y sanciones establecidas. Si la realidad fuese relativa, la mediación indagaría sobre la percepción que diferentes sujetos tienen de las causas, factores y demás aspectos y circunstancia que intervienen en los conflictos. La mediación en la resolución de conflictos requería recabar la opinión de las personas implicadas y asimismo favorecer las conversaciones entre ellas al objeto de que cada uno conozca la percepción del otro, y viceversa. Si la realidad es construida sociopráxicamente, se ha de saber cómo se percibe y construye la realidad, y, en tanto que la realidad construida es compatibilizada en redes en las que se participa, se ha de indagar sobre qué redes y realidades grupales compatibilizan una u otra realidades relacionadas con los factores, causas, motivaciones y aspectos varios que inciden en la construcción de la realidad conflictual. La mediación en este caso, ha de activar procesos participativos, en los que queden involucrados las diferentes redes y realidades grupales, con los que diseñar e implementar planes, programas y proyectos con los que anticiparse o/y resolver los conflictos.

En el ámbito educativo, los modelos predominantes de mediación no pueden afrontar problemáticas sociales como el bullying. Para abordas este tipo de problemática, el recurso a reglamentos, normas, derecho, deberes y sanciones, puede que no solo no resuelvan la situación, sino que la agraven aún más si los acosadores o/y sus adláteres responsabilizan de la sanción recibida a la(s) personas que son objeto del acoso. Para que la problemática se resuelva satisfactoriamente se han de involucrar en el mismo las múltiples redes y realidades grupales presentes en el centro escolar. Ello requiere conocer el tipo de relaciones que mantienen entre sí las diferentes realidades grupales presentes en el centro escolar, así como indagar sobre cómo se percibe y construye el otro. A tal fin se ha de poner en marcha un proceso participativo que elabore e implemente un Plan de Convivencia en el que se debata y se reflexiones sobre el reconocimiento del Otro, al objeto de construir un nosotros, esto es, un otro incluyente, en que cada uno, siendo diferente, con otros, forma conjunto.

\section{REFERENCIAS BIBLIOGRÁFICAS}

Bush, R. A., y Folger, J. (1996). La promesa de mediación: Cómo afrontar el conflicto mediante la revalorización y el reconocimiento. Barcelona: Ediciones Granica.

Calderón, P. (2009). “Teoría de Conflictos de Johan Galtung”, Revista Paz y Conflictos, N. 2. 60-81.

Castaneda, C. (1978). Una realidad aparte. México: Fondo de Cultura Económico.

Coob, S. (1993). "Empowerment and mediation: A narrative perpective", Negot, J9, 245- 259. DOI: https:// doi.org/10.1007/BF01000697

Cobb, S. (1997). "Una perspectiva narrativa en mediación", en Folger, J. y Jones, T. (Coords.), Nuevas direcciones en mediación: investigación y perspectivas comunicacionales (pp. 89-100). Buenos Aires: Paidós.

Cornelius, H., y Faire, S. (1995). Tú ganas, yo gano. Madrid: Gaia.

Diego de, R., y Guillén, C. (2006). Mediación: Proceso, tácticas y técnicas. Madrid: Pirámide.

Díez, F., y Tapia, G. (1999). Herramientas para trabajar en mediación. Buenos Aires: Paidós.

Fisher, R. y Ury, W. (1981). Getting to yes: Negotiating agreement without giving in. Boston: Houghton Mifflin.

Folger, J. y Jones, T. (1997). Nuevas direcciones en mediación: investigación y perspectivas comunicacionales. Buenos Aires: Paidós.

Freire, P. (1970). Pedagogía del oprimido. Montevideo: Tierra Nueva.

Galtung, J. (1998). Tras la violencia, 3R: reconstrucción, reconciliación, resolución: afrontando los efectos visibles e invisibles de la guerra y la violencia. Bilbao: Gernika Gogoratuz, 
Manuel Montañés Serrano, et al. LA RESOLUMEDiaCión de CONFLICTOS EN EL ÁMBITO EDUCATIVO DE MANERA ...

Glasersfeld, von E. (1994). "La construcción del conocimiento”, en Nuevos paradigmas, cultura y subjetividad. Buenos Aires: Paidós.

Max-Neeff, Elizalde y Hopenhayn (1986). Desarrollo a Escala Humana: una opción para el futuro. Barcelona: Icaria.

Montañés, M. (2006). “Diseño de una estrategia participativa conversacional con la que propiciar la formulación participada del plan local de inmigración”, en Encina, J. y Montañés, M. (Coord.). Construyendo colectivamente la convivencia en la diversidad. Los retos de la inmigración. Sevilla: UNILCO.

Montañés, M. (2009). Metodología y Técnicas participativas. Teoría y práctica de una estrategia de investigación participativa. Barcelona: UOC.

Montañés, M. (2012). “Una estrategia participativa conversacional con la que producir conocimiento y propuestas de actuación sociocultural", Revista de Antropologia Experimental, N. 12, 67-90.

Montañés, M. Martín, P. (2017). De la IAP a las Metodologías Sociopráxicas, Hábitat y Sociedad, N. $10,35-52$.

Montañes, M. y Ramos Muslera, EA. (2012). "La paz transformadora: una propuesta para la construcción participativa de paz y la gestión de conflictos desde una perspectiva sociopráxica”, Revista de Ciencias Sociales Obets, Vol. 7 , N. 2, 241-270.

Muldoon, B. (1998). El corazón del conflicto: del trabajo al hogar como campos de batalla, comprendiendo la paradoja del conflicto como camino hacia la sabiduría. Buenos Aires: Paidós.

Napoli di, P. (2016). "La violencia en las escuelas secundarias desde tres perspectivas de análisis. Hacia un estado del arte”, Zona Próxima, N. 24, 61-84. DOI: http://dx.doi.org/10.14482/zp.22.5832

Parkinson, L. (2012). Mediación familiar. Teoría y práctica: principios y estrategias operativas. Barcelona: Gedisa.

Prada, J., y López, A. (2005). "La mediación como estrategia de resolución de conflictos en el ámbito escolar", Documentación Social, N. 148, 99-116

Prigogine, I., y, Stengers, I. (1985). Order out of Chaos. Londres: Fontana.

Ramos, E. (2015). Paz Transformadora (y Participativa): teoría y método de la paz y el conflicto desde la perspectiva sociopráxica. Tegucigalpa: IUDPAS-UNAH.

Ramos, E. (2019). “Práxis participativa de la paz transformadora: teoría y método”. En Pérez de Armiño, K., y Zirión, I., (Coords.) Pax Crítica. Aportes teóricos a las perspectivas de paz posliberal. Madrid: Tecnos. 425-471.

Redorta, J. (2020). "Conflictos estructurales: elementos para intervención en conflictos crónicos", Revista Latinoamericana de Estudios de la Paz y el Conflicto, Vol. 1, N. 2, 92-110.

Torrego, J. C. (2001). "Modelos de regulación de la convivencia”, Cuadernos de pedagogía, N. 304, 22-28. 